ORIGINAL ARTICLE

\title{
Versican expression in pharyngeal squamous cell carcinoma: an immunohistochemical study
}

\author{
M J Pukkila, A S T Kosunen, J A Virtaniemi, E J Kumpulainen, R T Johansson, J K Kellokoski, \\ J Nuutinen, V-M Kosma
}

J Clin Pathol 2004;57:735-739. doi: 10.1136/icp.2003.014589

See end of article for authors' affiliations

Correspondence to:

Dr M Pukkila, Department of Otorhinolaryngology, Head and Neck Surgery, University of Kuopio, Kuopio University Hospital, PO Box 1777, FIN-70211, Kuopio, Finland:

Matti.Pukkila@kuh.fi

Accepted for publication 2 February 2004

\begin{abstract}
Aims: To study the expression of versican, a large proteoglycan involved in repressing adhesion between cells and the extracellular matrix in pharyngeal squamous cell carcinoma (PSCC), and its relation to the expression of p53 and catenins, histological differentiation, clinical data, and prognosis.

Methods: For the retrospective survey, primary tumours for analyses were obtained from 118 patients diagnosed with PSCC of the oropharynx or hypopharynx. The immunohistochemical expression of versican was studied and was related to the expression pattern of $\mathrm{p} 53$ and catenins, in addition to clinical data and survival.

Results: In the primary tumours, strong stromal versican expression was graded as low in $59(50 \%)$ and high in 59 (50\%) cases. In addition, intracellular versican staining was seen in nine (8\%) tumours. In local lymph node metastases, strong stromal versican staining was significantly more frequent compared with the primary tumours $(p=0.018)$. Strong stromal versican staining was more frequently seen in less advanced tumours $(p=0.015)$. There was no association between versican expression and the other investigated variables (p53, catenins, TNM status, and histological grade). Neither stromal nor intracellular versican expression predicted overall survival in these patients.

Conclusions: Versican was more strongly expressed in the stroma of local metastases and in the earlier stages of disease in PSCC. However, versican expression was not an independent prognostic factor in this entity.
\end{abstract}

$\mathrm{V}$ ersican, a large chondroitin sulfate proteoglycan, belongs to the aggrecan gene family, which also includes aggrecan, neurocan, and brevican. ${ }^{1}$ It is a component of the extracellular matrix (ECM) in various soft tissues, and the protein contains several active domains. At the N-terminal globular end (Gl), versican carries the hyaluronan (HA) binding domain, whereas at the C-terminal globular end (G3), contains one C-type lectin domain, two epidermal growth factor domains, and one complement regulatory domain. ${ }^{23}$ The central domain, to which the glycosaminoglycan (GAG) side chains are attached, is located between the G1 and G3 domains of the versican core protein. Transcriptional variations in combining the two possible subdomains ( $\alpha \mathrm{GAG}$ or $\beta \mathrm{GAG}$ ) of the GAG attachment region produce four different versican isoforms, namely: V0, V1, V2, and V3. ${ }^{2}$ The name versican highlights the diverse biological actions of this molecule: it has been shown to take part in cell adhesion, proliferation, migration, and assembly of the ECM. ${ }^{4}$ Physiological versican expression has been demonstrated in various adult tissues including smooth muscle, fibrous and elastic cartilage, the central and peripheral nervous system, glandular epithelia, blood vessels, and the skin. ${ }^{5}$ The high expression of versican reported in several malignant tumours-such as carcinomas of the gastrointestinal tract, ${ }^{6}$ breast, $^{78}$ ovary, ${ }^{9}$ and prostate, ${ }^{10}$ in addition to melanomas, ${ }^{11}$ mesotheliomas, ${ }^{12}$ sarcomas, ${ }^{13}$ and brain tumours ${ }^{14}$ - suggests that versican is involved in the development and progression of cancer. Versican may also accelerate cell proliferation, and the growth stimulatory effect of versican is thought to result from its destabilising effect on focal cell contacts, which then repress cell adhesion and promote tumour growth. ${ }^{12}{ }^{15-17}$ In epithelial tumour cell lines, excess versican production is associated with high mitotic activity in vitro. ${ }^{12}$ Versican mRNA (indicating versican gene activation) has recently been demonstrated both in tumour cells ${ }^{12}$ and in the tumour stroma. ${ }^{8}$ Peritumorous versican expression seems to be, at least in part, induced in stromal cells by factors secreted by carcinoma cells. ${ }^{78} 19$ Overall, increased stromal versican expression has been associated with more aggressive behaviour in several malignant tumours. $^{7} 9^{1014}$ Furthermore, in their recent study, Yoon et al demonstrated that the versican gene is regulated by the tumour suppressor p53 in vitro. ${ }^{20}$ The exact role of versican in human neoplasms still remains unclear.

"The name versican highlights the diverse biological actions of this molecule: it has been shown to take part in cell adhesion, proliferation, migration, and assembly of the extracellular matrix"

In the European Union, head and neck cancer (HNC) comprises $10 \%$ of all malignant tumours in men and $2 \%$ in women. ${ }^{21}$ The estimated age standardised annual incidence of HNC in Europe is 35.4/100 000 for men and 4.5/100 000 for women, respectively. ${ }^{21}$ Histologically, more than $90 \%$ of these tumours are squamous cell carcinomas (SCCs). ${ }^{22}$ The prognosis of patients with HNC varies substantially, depending on the tumour site: survival is the lowest in patients with oropharyngeal or hypopharyngeal tumours. ${ }^{23}$

We studied versican expression, which has not been explored previously in HNC, in a large cohort of pharyngeal SCC tumours (PSCCs). In addition, the interrelations

Abbreviations: $E C M$, extracellular matrix; GAG, glycosaminoglycan; $\mathrm{HA}$, hyaluronan; H\&E, haematoxylin and eosin; HNC, head and neck cancer; IHC, immunohistochemistry; OS, overall survival; PBS, phosphate buffered saline; PSCC, pharyngeal squamous cell carcinoma; SCC, squamous cell carcinoma 
between versican, p53, catenins, clinicopathological factors, and survival of patients were investigated to elucidate the role of versican in head and neck SCC.

\section{MATERIALS AND METHODS Study cohort and clinical data}

All patients in Kuopio University Hospital district in Eastern and Central Finland diagnosed with oropharyngeal or hypopharyngeal SCC between 1975 and 1998 were identified from the population based Finnish Cancer Registry and from hospital records. Twenty patients were excluded because of a lack of histological material, and 118 patients with sufficient tumour material for further histological investigations were studied. From previous investigations, catenin and p53 staining data were available for all patients. ${ }^{24} 25$ In an additional 14 cases, histological material from local lymph node metastases was available for analysis. Two otorhinolaryngologists (MJP and JAV) and one oncologist (EJK) reviewed all clinical data. The tumours were staged according to the Union Internationale Contre le Cancer (UICC) classification (1997), based on clinical otorhinolaryngological status, endoscopy, and chest $x$ ray. ${ }^{26}$ The Karnofsky performance status at the time of diagnosis was coded. ${ }^{27}$ All patients were regularly seen by an otorhinolaryngologist or oncologist until death or May 1999 and complete follow up data for each of the 118 patients was available.

\section{Histology}

The original haematoxylin and eosin (H\&E) stained sections from the 118 primary tumours and 14 local lymph node metastases were reviewed by an experienced pathologist (VMK) and the diagnosis of SCC was confirmed. The histological differentiation of the primary tumour was simultaneously re-evaluated and classified according to the World Health Organisation classification as good, moderate, or poor. $^{28}$

\section{Immunohistochemistry}

When reviewing the original H\&E stained sections, the most representative block was chosen, and cut into $5 \mu \mathrm{m}$ thick sections for immunohistochemistry (IHC). For versican, the paraffin wax embedded sections were incubated for 30 minutes at $58^{\circ} \mathrm{C}$, washed three times in xylene for seven minutes, a further three times in $99.5 \%$ ethanol, and twice in $96 \%$ ethanol, with each wash lasting for five minutes. Thereafter, sections were rinsed twice for three minutes in distilled water. Sections were heated in a microwave oven at $800 \mathrm{~W}$, for three five minute cycles in $0.01 \mathrm{~mol} /$ litre citrate buffer ( $\mathrm{pH}$ 6.0). The evaporated buffer was replaced with distilled water. Rehydrated sections were left standing for 18 minutes in the buffer, and were then washed twice for five minutes in phosphate buffered saline (PBS). Endogenous peroxidase activity was blocked with $5 \% \mathrm{H}_{2} \mathrm{O}_{2}$ in distilled water for five minutes. Sections were again washed three times in distilled water and twice in PBS, with each wash lasting for five minutes. After treatment for 35 minutes at room temperature with $1.5 \%$ normal horse serum (Vectastain Elite ABC kit; Vector Laboratories, Burlingame, California, USA) in PBS, monoclonal mouse antihuman versican (clone 2B1; Seikagaku Corporation, Tokyo, Japan) antibody, which recognises all human versican isoforms, ${ }^{13}$ was applied to the sections at a dilution of $1 / 500$ in $1.5 \%$ normal horse serum (Vectastain Elite ABC kit) and incubated for 20 hours at $4{ }^{\circ} \mathrm{C}$. Thereafter, the samples were washed twice for five minutes with PBS and the biotinylated secondary antibody was applied to the samples for 45 minutes at room temperature, after which it was washed twice for five minutes with PBS. Avidin-biotin peroxidase reagent (Vectastain Elite ABC kit) was applied to the samples for 50 minutes at room temperature, after which the samples were again washed twice for five minutes with PBS. The bound primary antibody was visualised by incubating the samples for five minutes in 0.05\% diaminobenzidine tetrahydrochloride (DAB; Sigma, St Louis, Missouri, USA) solution. The slides were rinsed in tap water for three minutes and in distilled water for two minutes, counterstained with Mayer's haematoxylin, dehydrated, cleared, and mounted with DePex (BDH, Poole, Dorset, UK). In each staining batch, dermal tissue samples with known strong stromal versican expression around normal hair follicles were used as positive controls, ${ }^{29}{ }^{30}$ and corresponding samples together with one tumour sample from the present series without the use of primary antibody as negative controls.

For the immunohistochemical demonstration of the expression of $\alpha, \beta$, and $\gamma$ catenins, in addition to p53 in the tumours, mouse anti- $\alpha$, anti- $\beta$, and anti- $\gamma$ catenin monoclonal antibodies (Transduction Laboratories, Lexington, Kentucky, USA) and mouse antihuman p53 (D07; Dako A/ S, Glostrup, Denmark) were used, as described previously. ${ }^{24} 25$

\section{Scoring of immunohistological staining}

To assess stromal versican expression, the total intratumorous and peritumorous stromal area was studied by two investigators (MJP and VMK) unaware of the clinical data, with a dual headed microscope. The proportion of the whole studied area that stained strongly was assessed on a continuous $(0-100 \%)$ scale, using the strong staining in the dermal positive control tissues as a standard. According to the median percentage ( $10 \%)$, the samples were divided into two categories-one with a low proportion of strong stromal versican expression $(<10 \%)$ and another with a high proportion of strong stromal versican expression $(\geqslant 10 \%) .{ }^{9}$ In addition, intracellular versican staining, seen in a few samples, was simultaneously graded as negative or positive. ${ }^{9}$ The expression of catenin on the plasma membrane was graded as normal or reduced (cutoff point, 90\%), nuclear $\beta$ catenin expression as negative or positive (cutoff point, $10 \%$ ), nuclear p53 expression as high or low, and cytoplasmic p53 expression as negative or positive, as reported previously. ${ }^{24}$

\section{Statistics and ethics}

The representativeness of the patients with tissue samples available for this study $(\mathrm{n}=118)$ of the whole patient group $(n=138)$ was checked with a non-parametric $\chi^{2}$ test for the categorical variables (sex, site, histological differentiation, stage, and Karnofsky performance status). For age (continuous variable) the representativeness was checked with the one sample $t$ test. The association between stromal versican staining patterns and clinicopathological variables was analysed with the $\chi^{2}$ test. Comparisons between the intensity of strong stromal versican staining in the primary tumours and local metastases were analysed with Wilcoxon's nonparametric test for two related samples (Monte Carlo simulation with 10000 samples).

The univariate analyses of overall survival (OS) were calculated according to the Kaplan-Meier method..$^{31}$ Cancer related OS was calculated from the date of primary diagnostic biopsy to the end of follow up or death. Only deaths from PSCC were included as events in the survival analyses. Multivariate OS analyses (Cox proportional hazards model) ${ }^{32}$ were performed in a stepwise manner (entry limit, $\mathrm{p}<0.05$; removal limit, $p \geqslant 0.1$ ). Baseline covariates used in the Cox proportional hazard model were age, sex, site (oropharynx or hypopharynx), histological differentiation (categorical, reference class good), $\mathrm{T}$ class (categorical, reference class $\mathrm{Tl}$ ), presence of lymph node metastases ( $\mathrm{NO}$ or $\mathrm{N}+$ ), distant metastases ( $\mathrm{M} 0$ or Ml), Karnofsky status $(\geqslant 70$ or $<70$ ), strongest stromal versican expression (high or low), 
intracellular versican expression (negative or positive), catenin expression (normal or reduced), nuclear $\beta$ catenin expression (negative or positive), nuclear p53 expression (high or low), and cytoplasmic p53 expression (negative or positive). In another test setting, stage was used as a categorical variable (reference class SI) instead of $\mathrm{T}$ class. SPSS for Windows Release 10.0 was used for statistical analysis.

The research was approved by the research ethical committee of Kuopio University and Kuopio University Hospital, and the Finnish Ministry of Social Affairs and Health granted permission for collecting data from the Finnish Cancer Registry and from hospital records. The authors had no conflicts of interests with relation to the study methodology, results, or other sectors of the study.

\section{RESULTS}

Table 1 summarises the patient and treatment data. The median age of the patients at the time of diagnosis was 63 (range, 36-89) years. The median follow up time was 43 (range, 1-332) months. The baseline characteristics (sex, age, Karnofsky performance status, histological differentiation, tumour site, and stage) of the patients with tissue samples available for versican IHC $(\mathrm{n}=118)$ did not differ from the original patient group $(\mathrm{n}=138)(\mathrm{p} \geqslant 0.54)$. In $36(29 \%)$ patients the tumour did not relapse. At the end of the follow up, only $19(15 \%)$ patients were alive; $78(63 \%)$ patients had died of PSCC and an additional $26(21 \%)$ patients had died of a non-PSCC related disease. In this last group, another malignant tumour caused death in eight patients. The most common treatment was radiotherapy alone. The primary treatment was considered curative in 104 and palliative in 11 patients. Three patients received basic care only because of their poor general condition.

In the normal tissue surrounding the tumours, the blood vessel walls and the peritumorous connective tissue were immunopositive for versican. No non-specific staining was present in the negative controls. Table 2 shows the immunohistological data with regard to versican expression. In the carcinomas, strong stromal versican expression was graded high (fig lA) in $59(50 \%)$ and low (fig lB) in $59(50 \%)$ primary tumours according to the median percentage $(10 \%)$. In addition, cytoplasmic versican staining was seen in nine $(8 \%)$ of the tumours (fig $1 C$ ). Intracellular versican accumulation was seen more frequently in tumours with high strong stromal versican expression, but the association was not significant. Of the 14 local lymph node metastases, strong stromal versican expression was graded as low $(<10 \%)$ in only one case, whereas in the remaining 13 cases it was graded as high ( $\geqslant 10 \%$ ) (fig lD). High strong stromal versican expression was significantly more common in local metastases than in the primary tumours $(\mathrm{p}=0.018$, Wilcoxon test for two related samples). A high percentage of strong versican staining was also more common in less advanced tumours (SI/II $v$ SIII/IV; $\mathrm{p}=0.015$ ) and in oropharyngeal tumours $(p=0.013)$. The nuclear p53 expression index was high in 43 (36\%) and low in 75 $(64 \%)$ of the tumours. In addition, the p53 protein was expressed in the tumour cell cytoplasm of 56 (47\%) tumours. No association between versican expression and the expression of p53 or the catenins, or other tested variables, was seen (data not shown).

In Kaplan-Meier univariate survival analyses, hypopharyngeal primary site $(p=0.032)$, high $\mathrm{T}$ class $(\mathrm{p}<0.0005)$, high tumour stage $(p<0.0005)$, and low Karnofsky performance status $(p<0.0005)$, in addition to nuclear $\beta$ catenin expression $(\mathrm{p}=0.0012)$, high nuclear $\mathrm{p} 53$ expression index $(\mathrm{p}=0.014)$, and cytoplasmic p53 expression $(\mathrm{p}=0.040)$ predicted worse OS. Neither stromal $(p=0.17)$ nor
Table 1 Summary of clinicopathological and treatment data $(n=118)$

\begin{tabular}{|c|c|}
\hline Variable & Number of patients (\%) \\
\hline \multicolumn{2}{|l|}{ Sex } \\
\hline Male & $89(75)$ \\
\hline Female & $29(25)$ \\
\hline Age, years (median) & 63 \\
\hline Median duration of symptoms (months) & 3 \\
\hline \multicolumn{2}{|l|}{ Site } \\
\hline Oropharynx & $75(64)$ \\
\hline Hypopharynx & $43(36)$ \\
\hline \multicolumn{2}{|l|}{ T class } \\
\hline $\mathrm{Tl}$ & $16(14)$ \\
\hline T2 & 40 (34) \\
\hline T3 & $22(18)$ \\
\hline $\mathrm{T} 4$ & $40(34)$ \\
\hline \multicolumn{2}{|l|}{$N$ class } \\
\hline NO & $68(58)$ \\
\hline N1 & $18(15)$ \\
\hline N2 & $29(24)$ \\
\hline N3 & $3(3)$ \\
\hline \multicolumn{2}{|l|}{$M$ class } \\
\hline MO & $113(96)$ \\
\hline M1 & $5(4)$ \\
\hline \multicolumn{2}{|l|}{ Stage } \\
\hline I & $9(8)$ \\
\hline ॥ & $25(21)$ \\
\hline III & $24(20)$ \\
\hline IV & $60(51)$ \\
\hline \multicolumn{2}{|l|}{ Histological differentiation } \\
\hline Good & $27(23)$ \\
\hline Moderate & $56(47)$ \\
\hline Poor & $35(30)$ \\
\hline \multicolumn{2}{|l|}{ Karnofsky performance status } \\
\hline 90 & $7(6)$ \\
\hline 80 & $18(15)$ \\
\hline 70 & $53(45)$ \\
\hline 60 & $25(21)$ \\
\hline 50 & $13(11)$ \\
\hline 40 & $2(2)$ \\
\hline \multicolumn{2}{|l|}{ Primary treatment } \\
\hline Radiotherapy & $75(64)$ \\
\hline Surgery & $5(4)$ \\
\hline Surgery and radiotherapy & $34(28)$ \\
\hline Chemotherapy & $1(1)$ \\
\hline No treatment & $3(3)$ \\
\hline \multicolumn{2}{|l|}{ Goal of the primary treatment } \\
\hline Curative & $104(88)$ \\
\hline Palliative & $11(9)$ \\
\hline No cancer specific treatment & $3(3)$ \\
\hline
\end{tabular}

intracellular versican expression $(p=0.18)$ was associated with OS in our patients. In the Cox proportional hazards model, only $\mathrm{T}$ class $(\mathrm{p}=0.0004)$, or alternatively stage $(p=0.003)$, Karnofsky performance status $(p=0.005)$, and nuclear $\beta$ catenin expression $(p=0.045)$ predicted worse OS.

\section{DISCUSSION}

We studied PSCC samples from all cases of oropharyngeal or hypopharyngeal SCC encountered in the Kuopio University Hospital district during the study period. In addition, the clinical data obtained for the analyses were complete and representative. Of the established prognostic factors, tumour size ( $\mathrm{T}$ class), the extent of the disease (stage), and the general condition of the patient (Karnofsky performance status) remained significant in multivariate analyses of survival, demonstrating the validity of our PSCC cohort for survival analyses.

Our present results show that in PSCC versican is expressed in tumour stroma and in the immediate vicinity of the tumour, which is in line with several previous reports. ${ }^{6-14}$ In malignant tumours, it may restrain cell adhesion and promote proliferation and invasion, in addition to migration. ${ }^{12}{ }^{17}$ 

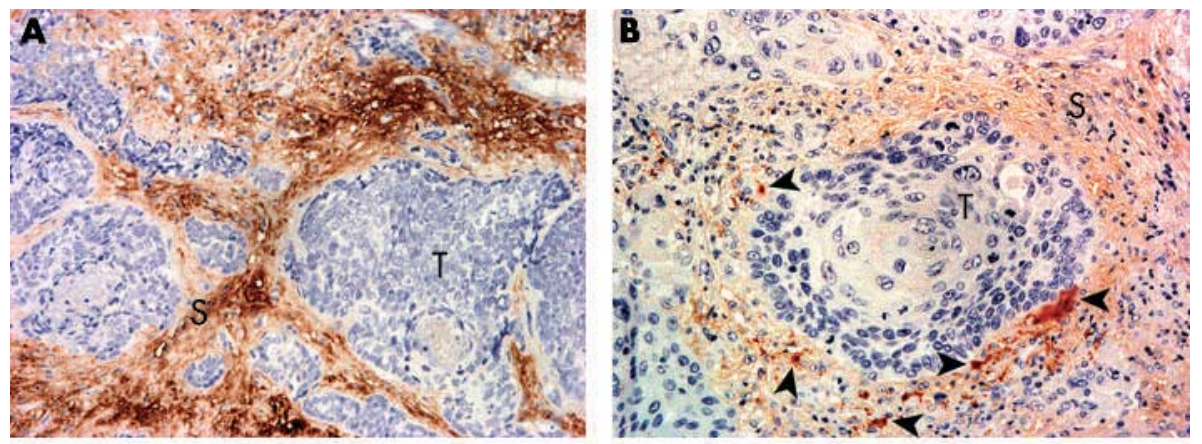

Figure 1 Immunohistochemical staining patterns found in pharyngeal squamous cell carcinoma, using Mayer's haematoxylin as a counterstain. (A) High strong stromal versican expression in a primary tumour; original magnification, $\times 200$. (B) Low strong stromal versican expression in a primary tumour; the arrowheads indicate scattered strong stromal versican expression; original magnification, $\times 200$. (C) Intracellular accumulation of versican (arrowheads) in cancer cells; original magnification, $\times 400$. (D) High strong stromal versican
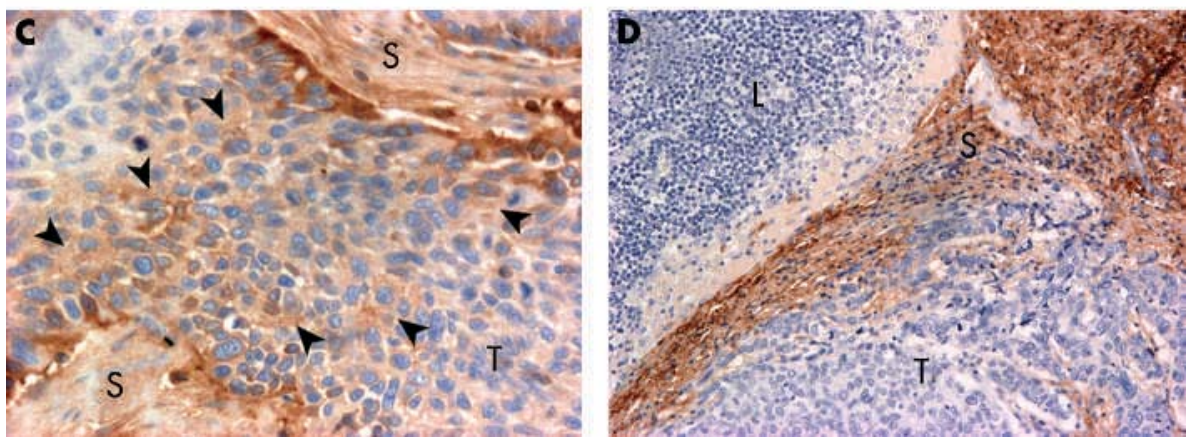
expression in lymph node metastasis; original magnification, $\times 200$ ). T, tumour cells; L, lymphatic cells; $S$, stroma.

It has been shown that versican accumulates both in tumour cells ${ }^{11}{ }^{12}{ }^{33}$ and in the tumour stroma. ${ }^{8}$ In the stroma, versican expression is also induced by factors secreted by the tumour cells. ${ }^{7819}$ In cancer, stromal versican has been shown to occur together with HA. ${ }^{9}$ The increased amounts of versican and associated molecules, such as polysaccharide HA, increase the extent of the pericellular matrix and thereby distend the ECM. ${ }^{4}$ Fibroblast proliferation can be induced by versican in vitro, ${ }^{15}$ and this might promote tumour stroma production in vivo. In malignant breast, prostate, and ovarian tumours, stronger stromal versican expression, as assessed by IHC, is associated with a more aggressive course of the disease..$^{7}{ }^{10}$ In gliomas, a similar trend has been suggested..$^{14}$ However, in the samples studied here versican expression had no prognostic significance. In clinical cancer research, similar inconsistencies between studies using IHC are common along with other biological factors. ${ }^{34}$

Interestingly, a significantly higher percentage of strong stromal versican expression was found in local metastases than in the primary tumours in PSCC. In breast carcinoma ${ }^{8}$ and melanoma, ${ }^{11}$ the expression of versican has also been reported in primary tumours and metastases. To our knowledge, differences in the intensity of versican expression between primary tumours and metastases have not been described previously. High versican expression in metastases in PSCC presumably reflects the importance of versican related mechanisms-that is, reduced adhesion, ${ }^{16}$ enhanced proliferation, ${ }^{15}{ }^{17}$ and migration ${ }^{16}$-in the formation and

Table 2 Summary of the expression of versican as assessed by immunohistochemistry $(n=118)$

\begin{tabular}{ll}
\hline Variable & Number (\%) \\
\hline $\begin{array}{l}\text { Strong stromal versican expression } \\
\text { Low }\end{array}$ & $59(50)$ \\
High & $59(50)$ \\
Intracellular versican expression & $109(92)$ \\
$\quad$ Negative & $9(8)$ \\
Positive & \\
\hline
\end{tabular}

growth of metastases. It may also indicate that versican is associated with the induction of angiogenesis and the production of intensely vascular stroma, which further promotes cancer invasion. ${ }^{85}$ The same mechanisms are probably responsible for the association between versican expression and aggressive tumour behaviour reported previously. ${ }^{7}{ }^{10} 14$ The formation of metastases can be considered to be a sign of aggressiveness, so that our finding of stronger versican expression in metastases suggests that induced stromal versican expression is associated with more aggressive behaviour also in PSCC. Furthermore, the stronger versican expression seen in less advanced tumours suggests that processes involving versican are particularly active in the earlier stages of PSCC.

\section{"To our knowledge, differences in the intensity of versican expression between primary tumours and metastases have not been described previously"}

In addition to stromal versican expression, clear intracellular expression in carcinoma cells was seen in some tumours, analogous to previous reports on cultured melanoma cells ${ }^{11}$ and highly proliferative mesothelioma and adenocarcinoma cell lines. ${ }^{12}$ Intracellular versican expression may be a consequence of altered production, storage, degradation, or cellular uptake of versican. However, the causes and mechanisms promoting intracellular versican expression require further investigations.

The roles of the tumour suppressor p53 in controlling genomic integrity and apoptosis of transformed cells are well established. ${ }^{36}$ In our earlier study, both nuclear and cytoplasmic p 53 overexpression were shown to be associated with a worse prognosis in PSCC. ${ }^{25}$ The in vitro results of Yoon et al show that versican gene expression is directly induced by p53. ${ }^{20}$ Therefore, using the immunohistochemical data we looked for interrelations between these two factors. However, at the protein expression level no significant associations (induction or suppression) were seen. This might be because of the mutation of p53 in carcinoma cells or because of several other confounding factors in vivo.

In addition, we have previously reported the expression of $\alpha, \beta$, and $\gamma$ catenins in PSCC. ${ }^{24}$ Because both versican and 
Take home messages

- Versican is expressed in the tumour stroma and occasionally in the tumour cell cytoplasm in pharyngeal squamous cell carcinoma (PSCC)

- The expression of versican is stronger in earlier stages of the disease and in lymph node metastases

- No association was found between the expression of versican and p53, as assessed by immunohistochemistry

- Versican was not an independent prognostic factor in PSCC

membranous catenins participate in cellular adhesion, we searched for signs of associations between versican and catenins. However, our results showed no interrelations between the expression of versican and either of the three catenins at the protein level in PSCC.

We conclude that in PSCC, versican is expressed in the tumour stroma and occasionally in the tumour cell cytoplasm. The expression is stronger in earlier stages of the disease and in metastases. However, we found no association between versican and p53 at the protein expression level. Finally, versican was not an independent prognostic factor in PSCC.

\section{ACKNOWLEDGEMENTS}

This study was funded by The Finnish Cultural Foundation of Northern Savo, Kuopio and Tampere University Hospital EVO Funds, The Northern Savo Cancer Fund, and The Finnish Cancer Foundation. We appreciate the constructive comments of Professor M Tammi and Senior Lecturer R Tammi in manuscript revision and the skilful immunohistochemical assistance of Mrs A Parkkinen, in addition to the expertise of Mrs P Halonen in statistical analyses and of $\mathrm{Mr}$ A Pelttari in photography.

\section{Authors' affiliations}

M J Pukkila, A S T Kosunen, J A Virtaniemi, J K Kellokoski, J Nuutinen, Department of Otorhinolaryngology, Head and Neck Surgery, University of Kuopio, Kuopio University Hospital, PO Box 1777, FIN70211, Kuopio, Finland

E J Kumpulainen, V-M Kosma, R T Johansson, Department of Oncology, University of Kuopio, Kuopio University Hospital

V-M Kosma, Department of Pathology and Forensic Medicine, University of Kuopio, Kuopio University Hospital, PO Box 1627, FIN-70211, Kuopio, Finland

V-M Kosma, Department of Pathology, University of Tampere, Tampere University Hospital, PO Box 2000, FIN-33521 Tampere, Finland

\section{REFERENCES}

1 Schwartz NB, Pirok EW 3rd, Mensch JR Jr, et al. Domain organization genomic structure, evolution, and regulation of expression of the aggrecan gene family. Prog Nucleic Acid Res Mol Biol 1999;62:177-225.

2 LeBaron RG, Zimmermann DR, Ruoslahti E. Hyaluronate binding properties of versican. J Biol Chem 1992:267:10003-10.

3 Zimmermann DR, Ruoslahti E. Multiple domains of the large fibroblast proteoglycan, versican. EMBO J 1989;8:2975-81.

4 Wight TN. Versican: a versatile extracellular matrix proteoglycan in cell biology. Curr Opin Cell Biol 2002;14:617-23.

5 Bode-Lesniewska B, Dours-Zimmermann MT, Odermatt BF, et al. Distribution of the large aggregating proteoglycan versican in adult human tissues. J Histochem Cytochem 1996;44:303-12.

6 Theocharis AD, Vynios DH, Papageorgakopoulou N, et al. Altered content composition and structure of glycosaminoglycans and proteoglycans in gastric carcinoma. Int J Biochem Cell Biol 2003;35:376-90.
7 Ricciardelli C, Brooks JH, Suwiwat S, et al. Regulation of stromal versican expression by breast cancer cells and importance to relapse-free survival in patients with node-negative primary breast cancer. Clin Cancer Res 2002:8:1054-60.

8 Brown LF, Guidi AJ, Schnitt SJ, et al. Vascular stroma formation in carcinoma in situ, invasive carcinoma, and metastatic carcinoma of the breast. Clin Cancer Res 1999;5:1041-56.

9 Voutilainen K, Anttila M, Sillanpää S, et al. Versican in epithelial ovarian cancer: relation to hyaluronan, clinicopathologic factors and prognosis. Int J Cancer 2003;107:359-64.

10 Ricciardelli C, Mayne K, Sykes PJ, et al. Elevated levels of versican but not decorin predict disease progression in early-stage prostate cancer. Clin Cancer Res 1998:4:963-71.

11 Touab M, Villena J, Barranco C, et al. Versican is differentially expressed in human melanoma and may play a role in tumour development. Am J Pathol 2002;160:549-57.

12 Gulyas M, Hierpe A. Proteoglycans and WT1 as markers for distinguishing adenocarcinoma, epithelioid mesothelioma, and benign mesothelium. J Pathol 2003; 199:479-87.

13 Isogai $Z$, Shinomura T, Yamakawa $N$, et al. $2 B 1$ antigen characteristically expressed on extracellular matrices of human malignant tumours is a large chondroitin sulfate proteoglycan, PG-M/versican. Cancer Res 1996;56:3902-8

14 Paulus W, Baur I, Dours-Zimmerman MT, et al. Differential expression of versican isoforms in brain tumours. J Neuropathol Exp Neurol 1996:55:528-33.

15 Zhang Y, Cao L, Yang BL, et al. The G3 domain of versican enhances cell proliferation via epidermal growth factor-like motifs. J Biol Chem 1998;273:21342-51

16 Ang LC, Zhang Y, Cao L, et al. Versican enhances locomotion of astrocytoma cells and reduces cell adhesion through its G1 domain. J Neuropathol Exp Neurol 1999;58:597-605.

17 Yang BL, Zhang Y, Cao L, et al. Cell adhesion and proliferation mediated through the Gl domain of versican. J Cell Biochem 1999;72:210-20.

18 Sakko AJ, Ricciardelli C, Mayne K, et al. Modulation of prostate cancer cell attachment to matrix by versican. Cancer Res 2003;63:4786-91.

19 Sakko AJ, Ricciardelli C, Mayne K, et al. Versican accumulation in human prostatic fibroblast cultures is enhanced by prostate cancer cell-derived transforming growth factor $\beta 1$. Cancer Res 2001;61:926-30.

20 Yoon H, Liyanarachchi S, Wright FA, et al. Gene expression profiling of isogenic cells with different TP53 gene dosage reveals numerous genes that are affected by TP53 dosage and identifies CSPG2 as a direct target of p53. Proc Natl Acad Sci U S A 2002;99:15632-7.

21 Black RJ, Bray F, Ferlay J, et al. Cancer incidence and mortality in the European Union: cancer registry data and estimates of national incidence for 1990. Eur J Cancer 1997;33:1075-107.

22 Slootweg PJ, Richardson M. Squamous cell carcinoma of the upper aerodigestive system. In: Gnepp DR, ed. Diagnostic surgical pathology of the head and neck. Philadelphia: WB Saunders, 2001:19-78.

23 Berrino F, Gatta G. Variation in survival of patients with head and neck cancer in Europe by the site of origin of the tumours. EUROCARE working group. Eur J Cancer 1998;34:2154-61.

24 Pukkila MJ, Virtaniemi JA, Kumpulainen EJ, et al. Nuclear $\beta$ catenin expression is related to unfavourable outcome in oropharyngeal and hypopharyngeal squamous cell carcinoma. J Clin Pathol 2001;54:42-7.

25 Pukkila MJ, Kumpulainen EJ, Virtaniemi JA, et al. Nuclear and cytoplasmic p53 expression in pharyngeal squamous cell carcinoma: prognostic implications. Head Neck 2002;24:784-91.

26 Pharynx. In: Sobin LH, Wittekind C, eds. TNM classification of malignant tumours, 5th ed. New York: Wiley-Liss, 1997:25-32.

27 Karnofsky DA, Abelmann WH, Craver LF, et al. The use of the nitrogen mustards in the palliative treatment of carcinoma. Cancer 1948;1:634-56.

28 Shanmugaratnam K. Histological typing of tumours of the upper respiratory tract and ear, 2nd ed. Berlin: Springer, 1991.

29 Zimmermann DR, Dours-Zimmermann MT, Schubert M, et al. Versican is expressed in the proliferating zone in the epidermis and in association with the elastic network of the dermis. J Cell Biol 1994;124:817-25.

30 Karvinen S, Kosma VM, Tammi MI, et al. Hyaluronan, CD44 and versican in epidermal keratinocyte tumours. Br J Dermatol 2003;148:86-94.

31 Kaplan EL, Meier P. Nonparametric estimation from incomplete observation. $J$ Am Stat Assoc 1958;53:457-81.

32 Cox DR. Regression models and life tables with discussion. Journal of the Royal Statistical Society Series B Methodological 1972;34:187-92.

33 Touab M, Arumi-Uria M, Barranco C, et al. Expression of the proteoglycans versican and mel-CSPG in dysplastic nevi. Am J Clin Pathol 2003; 1 19:587-93.

34 van den Brekel MWM, Bindels EMJ, Balm AJM. Prognostic factors in head and neck cancer. Eur J Cancer 2002;38:1041-3.

35 Banerjee AG, Liu J, Yuan Y, et al. Expression of biomarkers modulating prostate cancer angiogenesis: differential expression of annexin II in prostate carcinomas from India and USA. Mol Cancer 2003;2:34.

36 Bargonetti J, Manfredi JJ. Multiple roles of the tumour suppressor p53. Curr Opin Oncol 2002;14:86-91. 